\title{
Peritoneal Dialysis Is an Option for Acute Kidney Injury Management in Patients with COVID-19
}

\author{
José M. Rodríguez-Chagolla ${ }^{a}$ Enzo Vásquez Jiménez ${ }^{b}$ Leticia Herrera Arellano ${ }^{a}$ \\ Alberto Villa Torres $^{a}$ Nayeli Acosta García ${ }^{a}$ Dolores Aleman Quimbiulco ${ }^{a}$ \\ Sergio Armeaga Aguilar ${ }^{a}$ Magdalena Madero ${ }^{b}$ \\ ${ }^{a}$ Nephrology Department, Centro Médico Issemym Toluca, Toluca de Lerdo, Mexico; ${ }^{b}$ Nephrology Department, \\ Instituto Nacional de Cardiología Ignacio Chávez, Ciudad de México, Mexico
}

\section{Keywords}

COVID-19 disease · Peritoneal dialysis · Acute kidney injury

\begin{abstract}
In December 2019, cases of acute respiratory illness of unknown origin were reported in Wuhan, China. The disease is caused by "severe acute respiratory syndrome coronavirus 2 ". After identifying severe lung damage, injury to other organs, such as the kidney, has been identified. Peritoneal dialysis is a renal replacement therapy (RRT) and is at least as effective as other extracorporeal therapy options, with significant cost-effective advantages. However, this strategy is rarely used for the management of acute kidney injury in severe lung disease. In this review, we explore PD as an RRT strategy that may be a key instrument in countries and hospitals with limited access to all RRTs.

(c) 2020 S. Karger AG, Basel
\end{abstract}

\section{Introduction}

In December 2019, cases of severe acute respiratory syndrome of unknown origin were reported in Wuhan, in the Hubei province of China $[1,2]$. The disease was fi- nally shown to be caused by "severe acute respiratory syndrome coronavirus 2" (SARS-CoV-2) [3]. The World Health Organization recognized this disease as a pandemic, and by April 2020, cases have been reported in 211 countries, with more than 1.9 million confirmed cases [4]. In Mexico, about 256,848 cases have been diagnosed in all of the states [5]. Since the beginning of the epidemic, kidney injury associated with COVID-19 disease has been documented, in percentages as high as 15\% [6].

However, the available data suggest that the prevalence of acute kidney injury (AKI) in patients with $\mathrm{CO}$ VID-19 is variable. In another cohort study $(n=1,099)$, $5.3 \%$ of patients required admission to the intensive care unit (ICU) and only $0.5 \%$ of patients had AKI [7].

There are 3 proposed mechanisms for kidney injury that result from direct damage by cytokines (cytokine release syndrome, increased cytokine production, and hemophagocytic syndrome), organ crosstalk (heart disease or viral myocarditis, alveolar damage, rhabdomyolysis, elevation of peak airway pressure, and intra-abdominal hypertension), and systemic complications (volume overload, endotoxins, endothelial damage, loss of fluid into the interstitial space, and hypotension) [8]. The hypothesis of damage induced by inflammatory cytokines points to the systemic inflammatory state that karger@karger.com

(C) 2020 S. Karger AG, Basel

www.karger.com/bpu

Karger!
José M. Rodríguez-Chagolla

Universidad Nacional Autónoma de México, Nephrology and Internal Medicine Ave. Baja Velocidad KM. 57.5, S

Toluca de Lerdo 52170 (Mexico)

jm.rodriguez.chagolla@gmail.com 
originates from the infection itself and from acute tubular necrosis, as well as to direct injury associated with the presence of the virus in the kidney. This is because previously, the angiotensin-converting enzyme and dipeptidyl peptidase- 4 were identified as areas of junction for SARS-CoV and MERS-CoV [9, 10]. Unpublished data suggest that SARS-CoV-2 can directly infect tubular epithelium and induce acute kidney damage due to the presence of direct cytotoxicity and may promote CD68 macrophage activity, in addition to C5bC9 complement deposits that act as mediators of tubular injury. Other studies found a direct parenchymal infection of tubular epithelial cells and podocytes with marked acute tubular injury and erythrocyte aggregation occurs in severe lethal COVID-19 [11]. On the other hand, COVID-19 RNA has been found in the plasma of $15 \%$ of patients through real-time PCR [12]. This test also demonstrated that COVID-19 RNA is found in up to 91.7\% of AKI patients [13].

\section{Access to Renal Replacement Therapies in AKI for COVID-19 Disease in Developing Countries}

The presence of a greater number of AKI cases in patients with COVID-19 disease is a matter of debate. Some case series did not demonstrate an increased presence of AKI cases in patients with COVID-19 disease. They report that SARS-CoV-2 infection does not produce AKI and does not worsen the existing state of CKD in patients with COVID-19 disease [14]. However, these studies may not reflect possible scenarios in populations with a higher prevalence of obesity, diabetes, and CKD.

In the absence of established drugs or vaccines for severe COVID-19 disease, there is a pathophysiological justification for the implementation of therapies such as direct hemoperfusion, plasma adsorption on resin, continuous kidney replacement therapy (CKRT), and high doses of CKRT with MCO or HCO8 membranes. However, in developing countries, access to renal replacement therapies (RRTs) such as CKRT is limited and clinicians may find themselves in scenarios not necessarily similar to those described in developed countries.

Access to renal function replacement therapies in low- and lower middle-income countries (LLMICs, gross national income $<$ USD 4035) is known to be often scarce, with variations observed in regions of the same country or different areas of the same city. In these countries, lack of resources is the main reason for not providing dialysis therapies. While CKRT is used in approximately $30 \%$ of
AKI patients in high-income and upper-middle-income countries, less efficient alternative therapies are used more frequently in LLMICs (22\%). Paradoxically, and despite its value in low-resource settings, PD treatment is infrequent, both in LLMICs (7\%) and in high-income and upper-middle-income countries (3\%) [15]. Although PD was the first RRT strategy in the management of AKI [16], there is little evidence on its use in the current COVID-19 pandemic as an RRT strategy in the treatment of AKI.

\section{Efficacy of Peritoneal Dialysis in the Management of AKI}

The efficacy of PD in the management of AKI is at least as efficient as hemodialysis (HD) and possibly as efficient as hemodiafiltration $[17,18]$. Currently, the best evidence is found in a meta-analysis from Liu et al. [19] (analysis of 6 studies with 484 patients), where PD therapy and extracorporeal RRT are compared for management of AKI. In this study, PD therapy has little or no difference with other extracorporeal therapies concerning all-cause mortality and recovery of kidney function (Moderate level of evidence). On the other hand, PD may be slightly less effective in the removal of ultrafiltration volume (low level of evidence) and has no difference for infectious complications (low level of evidence). The efficacy of the correction of acidosis or Kt/V dosage may be higher in extracorporeal therapies; however, the evidence for this argument is very low [19].

A randomized study of patients with $\mathrm{AKI}$ and multiorgan failure admitted to the ICU that required RRT evaluated the outcomes of 125 patients assigned to RRT modalities in the continuous veno-venous hemodiafiltration mode $(N=62)$ and tidal peritoneal dialysis (TPD) $(N=63)$. The primary outcome of the study was that survival in patients at 28 days treated with TPD, compared to continuous veno-venous hemodiafiltration ( 69.8 vs. $46.8 \%, p<0.01$ ), is superior. Infectious complications were significantly lower and other outcomes such as the recovery of kidney function (60.3 vs. $35.5 \%$ ) were higher in the TPD group [20]. Other clinical trials that compare extracorporeal therapies versus $\mathrm{PD}$ for AKI management indicate that, in regards to the correction of uremia, acidosis, fluid overload, and hyperkalemia, results are not different between the groups. Nonetheless, the cost-benefit advantage is more favorable for the PD group [17]. 


\section{Peritoneal Dialysis in Patients with Acute Lung Injury or Severe Pneumonia}

It is believed that the elevation of intraperitoneal pressure (IPP) generated by PD may alter diaphragmatic movement, thereby decreasing pulmonary compliance, which could hinder the mechanical ventilation management and worsen respiratory failure [21-23]. This argument does not have solid scientific evidence; Vieira et al. [22] evaluated the effects of AKI while weaning from mechanical ventilation in a retrospective observational study. The RRT used in this study for all patients was HD, and the possible effects of PD on ventilator dynamics are not mentioned [22]. Almeida et al. [24] studied the effect of PD on mechanical ventilation in patients with AKI and evaluated 106 patients treated with RRT [24], in PD mode, and 80 with $\mathrm{HD}$. The evaluation of respiratory mechanics showed improvement in lung compliance after 3 PD sessions and resistance of the respiratory system (Rsr) remained without significant changes. On the other hand, an increase in the IPP after the first dialysate infusion was observed, but this became normalized in subsequent draining. Oxygenation parameters, like $\mathrm{FiO}_{2}$, were the same during the first and second $\mathrm{PD}$ sessions and decreased after the second session. $\mathrm{PaO}_{2} / \mathrm{FiO}_{2}$ progressively increased after the first PD session, and the analysis adjusted for the presence or absence of chronic obstructive pulmonary disease, lung infection, and mechanical ventilation time showed no differences from those previously described [24]. A recent study compared the effect of PD versus HD on the respiratory mechanics of patients with AKI; the prospective cohort study evaluated 154 patients, 37 on PD and 94 on HD. Researchers analyzed pulmonary static compliance, Rsr, and the oxygenation index for 3 days and evaluated patients at 3 separate times, 1, 2, and 3 (pre and post dialysis). The results showed that pulmonary static compliance increased, with no differences between the groups, Rsr remained stable among the patients with continuous PD and decreased among the HD patients, and oxygenation index increased in both groups. The authors conclude there is improvement in ventilatory mechanics in both groups, without significant differences [25].

\section{Clearance of Inflammatory Cytokines in Peritoneal Dialysis}

The proposed pathophysiological mechanisms for kidney injury related to COVID-19 disease, based on direct cytokine damage [8], may make the usage of addi- tional treatments, such as extracorporeal techniques or the so-called blood purification techniques, appealing. These techniques include (but are not limited to) hemofiltration, hemoperfusion, intermittent or continuous high volume hemofiltration, plasmapheresis, or adsorption [26].

The clearance of inflammatory cytokines in PD has been demonstrated in experimental models. Altmann et al. [27] proved that circulating interleukin 6 (IL-6) is eliminated through PD. Recombinant human IL-6 (RH IL-6) was administered intravenously to wild-type mice with $\mathrm{AKI}$ and then with $\mathrm{PD}$ was performed in high doses. RH IL-6 was determined in the liquid from PD, and high levels of RH IL-6 were found. Similarly, levels constantly decreased as the treatment progressed. Importantly, there is no crossed reaction between endogenous murine IL-6 and RH IL- 6 by ELISA, which indicates that circulating IL-6 enters the peritoneal dialysis fluid and may be effectively removed [27].

The adequacy of PD in removing inflammatory substances has been scarcely studied in humans. Zhao et al. [28]. evaluated the effectiveness of PD for the removal of inflammatory toxins. The results of the study showed that inflammatory factors, including TNF- $\alpha$, IL-6, and PCT, decreased significantly after treatment compared to pretreatment. They concluded that PD is effective for the removal of these substances [28]. Other authors have evaluated the efficacy of PD for cytokine clearance in pediatric populations, specifically in the cytokine-mediated capillary leak syndrome after cardiopulmonary bypass (CPB). Measurement of cytokine levels by ELISA in blood plas$\mathrm{ma}$, and peritoneal fluid (PF) from samples of 18 neonates, showed a significant increase of serum concentrations of IL-6, IL-8, and IL-10 after CPB. The study also found that concentrations of IL- 6 and IL- 8 in PF exceeded serum concentrations, while IL-10 concentrations were higher in serum. They concluded that PF may be a repository for harmful inflammatory cytokines after CPB, and removal of PF by PD could reduce serum cytokine concentrations [29].

\section{Peritoneal Dialysis in Pronation Strategies}

One of the strategies to improve oxygenation in patients with acute respiratory distress syndrome (ARDS) is to use the prone position, which favors the decrease of the pleural pressure gradient in the dependent regions and reduces arteriovenous shunts. It promotes an equitable distribution air and ventilation/perfusion ratio as well [30]. 
The utility of PD in the management of AKI in patients with ARDS in the pronation position has seldom been studied. This is in part because it is thought that increasing IPP may cause a decrease in lung capacity. When IPP is greater than $18 \mathrm{~cm} \mathrm{H}_{2} \mathrm{O}$, vital lung capacity decreases by more than $20 \%$. However, this IPP threshold can be achieved with multiple small volume cycles [31].

Klisnick et al. [32] reported a case of a 38-year-old patient with CKD and neurogenic pulmonary edema, treated with mechanical ventilation, in the prone position, and automated PD as RRT. This treatment achieved $1 \mathrm{~L}$ of ultrafiltration per day and $11.3 \mathrm{~mL} / \mathrm{min} / 1.73 \mathrm{~m}^{2}$ of $\mathrm{Cr}$ clearance. The prescription consisted of $1.5 \mathrm{~L}$ cycles with glucose bags of $1.36-3.86 \%$, an IPP $<18 \mathrm{~cm} \mathrm{H}_{2} \mathrm{O}$ was monitored, and $40 \mathrm{~L}$ of dialysate per day was achieved. Although they observed an increase in pulmonary capillary wedge pressure due to the prone position, the infusion of the dialysis liquid was not affected [32]. Experience by Maryanne Y. Sourial and cols, describes 16 patients with $\mathrm{PD}$ who required pronation, in whom $\mathrm{PD}$ cycles were suspended while the patients were in the prone position, and they received HD or CKRT as supplementary treatment (if possible and depending on the location of the patient). After returning to the supine position, the PD replacements were restarted [33].

\section{Experience in PD in the Management of AKI Associated with COVID-19}

Evidence of PD in the management of AKI during the COVID-19 pandemic is limited. However, Maryanne Y. Sourial's group at the medical center in the Bronx, NY, refer to the need to start an urgent peritoneal dialysis program, due to the high demand for dialysis and shortages of personnel and supplies. The experience of this group indicates that $\mathrm{PD}$ is a viable strategy for $\mathrm{HD}$ in conditions of critical resource scarcity. These authors describe the results obtained in 30 patients with RRT requirement, reporting a mortality of $47 \%$. The success of PD treatment was achieved in $63 \%$ of patients. However, there were significant difficulties such as lack of training of nursing personnel and availability of supplies, as well as lack of adequate clearance and ultrafiltration for patients in UCI, in addition to half of the patients requiring pronation strategies, which limit PD therapy for long periods of time [33].

Preliminary data from other medical groups have been discussed in online forums, such as that presented on April 23, 2020, by the International Society for Peritoneal
Dialysis (ISPD)/International Society of Nephrology webinar "Use of PD for COVID-19-associated AKI: clinical experience and updated 2020 ISPD guidelines," where PD was defined as a potentially effective and safe strategy in the management of AKI, with advantages over CKRT strategies as a reduction in workload for staff [34].

\section{PD Catheter Insertion Techniques during the COVID-19 Pandemic}

In an environment where optimizing resources is relevant, increasing availability of PD patients, percutaneous catheter insertion with and without image guidance can be performed at bedside by surgeons' interventional radiologists and nephrologists and may be considered for peritoneal access [35]. Medical groups have derived this practice during the COVID-19 crisis, which affects the installation of catheter placement at the bedside of patients by transplant surgeons in ICU patients and by interventional radiologists using fluoroscopic guidance in non-ICU and nonintubated patients. They report the use of the PD catheter immediately after insertion, with low volumes of $1-1.5 \mathrm{~L}$ the first $24 \mathrm{~h}$, with subsequent increase to volumes greater than $2-2.5 \mathrm{~L}$ [33].

The other experiences on inserting a PD catheter into the patient's bedside come from data reported on April 23,2020 , by the ISPD/International Society of Nephrology webinar "Use of PD for COVID-19-associated AKI: clinical experience and updated 2020 ISPD guidelines," where Hugh Cairns and Elaine Bowes at Kings College Hospital, London, UK, describe that insertion of the PD catheter is performed by a senior nurse or by the consulting nephrologist; 27 of 32 attempts were successful [34]. Mika Nagatomo's group described PD catheter insertion to the rectovesical pouch using a portable $\mathrm{X}$-ray machine at the bedside and infused the peritoneal dialysate [36].

Data prior to the COVID-19 pandemic mention that the insertion of the PD catheter can be performed by percutaneous techniques guided or not guided by radiological techniques and procedures that can be performed by interventional radiologists, surgeons, and nephrologists. Other viable techniques are minilaparotomy in the patient's bed [35]. When compared to open procedures, percutaneous catheter placement with image guidance turns out as a cost-effective and safe procedure, including urgent-star PD [37]. Al Hwiesh described a percutaneous bedside placement of PD catheters without image guidance in 40 catheters in 38 patients. Exit-site infection occurred in $5 \%$ at month, and early exit-site leakage was 
observed in only 1 catheter; bowel perforation or serious hemorrhage was not reported. Catheter survival was $95 \%$ at 6 months and $87.5 \%$ at 12 months [38]. Like any other procedure, prophylactic antibiotic should be used at the time of PD access insertion according to ISPD guidelines [39].

There are recommendations during the surgical procedure that could minimize the risk of leak, such as the use of a cord suture to secure the deep cuff, which should be placed in the rectus muscle, and a paramedic over a midline incision in the peritoneal cavity [35]. In patients who have anticoagulation, it is always preferable to avoid vascular access when possible, so PD may be a suitable alternative. Therefore, placement of a peritoneal access should be minimally invasive and preferably image guided. Even in some protocols, heparin is instilled in the bags to decrease the clot formation due to thrombotic risk [33]. The best practices consensus protocol by interventional radiologists recommends removing anticoagulants 5 days before the procedure [37].

\section{Management of Peritoneal Fluid in Patients with COVID-19 Disease}

The recommendations for the management of peritoneal effluent in patients with COVID-19 disease include doing nothing more than what is usual practice and adding chlorine solution in a ratio of $500 \mathrm{mg} / \mathrm{L}$ for $1 \mathrm{~h}$ into the toilet before discharging remains. It is recommended to avoid the splashing of drops that may result from pressing the toilet discharge button [40].

\section{Insights of Peritoneal Dialysis in COVID-19 Pandemic}

Continuous PD is a form of CKRT and may be used in critically ill patients. The efficacy of PD in the management of AKI is at least as efficient as HD and possibly as efficient as hemodiafiltration $[17,18]$; however, in conditions of high morbidity as in current pandemic conditions, this strategy may not make a difference with other extracorporeal therapies concerning all-cause mortality and recovery of kidney function [19]. This includes patients with severe lung disease [25], such as that seen in COVID-19 disease. There is no evidence to support that pulmonary dynamics are severely altered by FP in the abdominal cavity [24]. Conversely, some studies support the fact that lung mechanics improve in a similar way to other RRT strategies [25].

Peritoneal Dialysis in AKI by COVID-19
The possibility of a prolonged pandemic poses the threat of equipment shortages and supply chain deficiencies [41]. In countries with scarce economic resources, access to extracorporeal RRT is limited and in many cases this alternative does not exist. In these vulnerable areas, PD may have clear advantages, over the need for minimally trained personnel, as well as the essential infrastructure required to offer treatment [17]. Another advantage is that in most of these countries, there is extensive experience for the employment of PD strategies [15]. Interestingly, the information available on the experience of this strategy in the management of AKI comes from high-resource countries, which means that in conditions of extreme morbidity, such as the COVID-19 pandemic, PD is useful and effective as a dialysis modality [33-35].

Strategies for prescribing PD in AKI are widely distributed (peritoneal dialysis for AKI, according to ISPD guidelines). The recommendation is to look for a weekly $\mathrm{Kt} / \mathrm{V}$ urea of 3.5, which provides results comparable to those of daily $\mathrm{HD}$, albeit this dose may not be necessary for all patients, and a lower goal of weekly Kt/V close to 2.1 may be acceptable [42]. Probably in the COVID-19 crisis, continuous APD would be the preferred modality because it minimize the number of connections and disconnections; also, fewer changes can be made to prescriptions, monitoring electrolyte disturbances. However, the available modality will always be the best. Unfortunately, APD machines are not available in some places in developing countries [35].

Recently, Ponce et al. [21] published PD prescription recommendations, which highlight the strategies with short cycles for patients with severe hyperkalemia, metabolic acidosis, fluid overload, or uremic symptoms, for which the modalities of HVPD or PD of tide would be the modalities of choice, in this case the total volume of dialysate must reach 30-36 L [43]. Strategies such as tidal $\mathrm{PD}$ may decrease patient monitoring time and therefore lessen exposure in medical personnel. Given the lack of evidence to predict the behavior of $\mathrm{PD}$ in patients with ARDS, the measurement of IPP may help to estimate the volume of $\mathrm{PF}$, with a maximum permissible volume for an IPP of $18 \mathrm{~cm} \mathrm{H}_{2} \mathrm{O}$ [44].

Expert recommendations mention that PD can be started early, before patients develop ARDS. However, when ARDS occurs, PD should not be used as the first option for RRT [43]. Regarding patients requiring pronation, there are few reports of cases where PD was used successfully $[31,32]$; however, there is insufficient information to recommend PD when the patient is in this po- 
sition. Therefore, other RRT strategies should be considered [43], mainly due to the resulting technical problems and possible difficulties in handling the mechanical ventilator [35].

\section{Conclusion}

Peritoneal dialysis is a modality of RRT at least as efficient as other extracorporeal RRT options with important cost-effective advantages, making it a key instrument in countries without access to all RRTs. There is no theoretical justification for it to be not used in patients with severe acute respiratory syndrome as those observed in COVID-19 disease. The prescription of PD in severe lung disease must be adapted to each patient specifically, according to international guidelines. Complementary studies are required to establish clear management guidelines in this group of patients.

\section{Conflict of Interest Statement}

The authors declare no conflicts of interests.

\section{Funding Sources}

This research was conducted without sponsorship.

\section{Author Contributions}

Rodríguez-Chagolla José [1]: first author, Department of Nephrology of the Centro Médico Issemym Toluca, México. Magdalena Madero [2]: medical researcher, National Institute of Cardiology Ignacio Chávez, México. Enzo Vásquez Jiménez [2], Department of Nephrology of the National Institute of Cardiology, fellow. Herrera Arellano Leticia, Department of Nephrology of the Centro Médico Issemym Toluca, México. Armeaga Aguilar Sergio, Department of Nephrology of the Centro Médico Issemym Toluca, México. Villa Torres José, Department of Nephrology of the Centro Médico Issemym Toluca, México, fellow. Acosta García Nayeli, Department of Nephrology of the Centro Médico Issemym Toluca, México, fellow. Aleman Quimbiulco Dolores, Department of Nephrology of the Centro Médico Issemym Toluca, México, fellow.

\section{References}

1 Li Q, Guan X, Wu P, Wang X, Zhou L, Tong $\mathrm{Y}$, et al. Early transmission dynamics in $\mathrm{Wu}-$ han, China, of Novel Coronavirus-infected Pneumonia. N Engl J Med. 2020 Mar 26; 382(13):1199-207.

2 Zhu N, Zhang D, Wang W, Li X, Yang B, Song $\mathrm{J}$, et al. China Novel Coronavirus investigating and research team. A Novel Coronavirus from patients with Pneumonia in China, 2019. N Engl J Med2020 Feb 20;382(8):727-33.

3 Lu R, Zhao X, Li J, Niu P, Yang B, Wu H, et al. Genomic characterisation and epidemiology of 2019 novel coronavirus: implications for virus origins and receptor binding. Lancet. 2020 Feb 22;395(10224):565-74.

4 World Health Organization (WHO). Coronavirus disease 2019 (COVID 19) situation report-78. 2020. : https://www.who.int/docs/ default-source/coronaviruse/situationreports/20200407-sitrep-78-covid-19. pdf?sfvrsn = bc43e1b_2.

5 Ministry of health of Mexico. New Coronavirus worldwide technical report (COVID-19). 2020. : https://covid19.sinave.gob.mx/.

6 Zhou F, Yu T, Du R, Fan G, Liu Y, Liu Z, et al. Clinical course and risk factors for mortality of adult inpatients with COVID-19 in Wuhan, China: a retrospective cohort study. Lancet. 2020 Mar 28;395(10229):1054-62.

7 Guan WJ, Ni ZY, Hu Y, Liang WH, Ou CQ, $\mathrm{He}$ JX, et al. Clinical characteristics of coronavirus disease 2019 in China. N Engl J Med. 2020 Feb 28.
8 Ronco C, Reis T. Kidney involvement in COVID-19 and rationale for extracorporeal therapies. Nat Rev Nephrol. 2020 Apr 9.

9 Li W, Moore MJ, Vasilieva N, Sui J, Wong SK, Berne MA, et al. Angiotensin-converting enzyme 2 is a functional receptor for the SARS coronavirus. Nature. 2003 Nov 27;426(6965): 450-4.

10 Raj VS, Mou H, Smits SL, Dekkers DH, Müller MA, Dijkman R, et al. Dipeptidyl peptidase 4 is a functional receptor for the emerging human coronavirus-EMC. Nature. 2013 Mar 14;495(7440):495251-4

11 Su H, Yang M, Wan C, Yi LX, Tang F, Zhu $\mathrm{HY}$, et al. Renal histopathological analysis of 26 postmortem findings of patients with $\mathrm{CO}$ VID-19 in China. Kidney Int. 2020 Apr 9.

12 Huang C, Wang Y, Li X, Ren L, Zhao J, Hu Y, et al. Clinical features of patients infected with 2019 novel coronavirus in Wuhan, China. The Lancet. 2020 Feb 15;395(10223):497506.

13 Chu KH, Tsang WK, Tang CS, Lam MF, Lai FM, To KF, et al. Acute renal impairment in coronavirus-associated severe acute respiratory syndrome. Kidney Int. 2005 Feb;67(2): 698-705.

14 Wang L, Li X, Chen H, Yan S, Li D, Li Y, et al. Coronavirus disease 19 infection does not result in acute kidney injury: an analysis of 116 hospitalized patients from Wuhan, China. Am J Nephrol. 2020 Mar 31:1-6.
15 Mehta RL, Burdmann EA, Cerdá J, Feehally J, Finkelstein F, García-García G, et al. Recognition and management of acute kidney injury in the International Society of Nephrology 0by25 Global Snapshot: a multinational cross-sectional study. Lancet. 2016 May 14; 387(10032):2017-25.

16 Maxwell MH, Rockney RE, Kleeman CR, Twiss MR. Peritoneal dialysis. 1. Technique and applications. J Am Med Assoc. 1959; 170(8):917-24.

17 George J, Varma S, Kumar S, Thomas J, Gopi S, Pisharody R. Comparing continuous venovenous hemodiafiltration and peritoneal dialysis in critically ill patients with acute kidney injury: a pilot study. Perit Dial Int. 2011; 31(4):422-9.

18 Gabriel DP, Caramori JT, Martim LC, Barretti P, Balbi AL. High volume peritoneal dialysis vs daily hemodialysis: a randomized, controlled trial in patients with acute kidney injury. Kidney Int Suppl. 2008;108(108):S8793.

19 Liu L, Zhang L, Liu GJ, Fu P. Peritoneal dialysis for acute kidney injury. Cochrane Database Syst Rev. 2017 Dec 4;12:CD011457.

20 Al-Hwiesh A, Abdul-Rahman I, Finkelstein F, Divino-Filho J, Qutub H, Al-Audah N, et al. Acute kidney injury in critically Ill patients: a prospective randomized study of tidal peritoneal dialysis versus continuous renal replacement therapy. Ther Apher Dial. 2018 Aug; 22(4):371-9. 
21 Ponce D, Gobo-Oliveira M, Balbi AL. Peritoneal dialysis treatment modality option in acute kidney injury Blood Purif. 2017;43(13):173-8.

22 Vieira JM Jr, Castro I, Curvello-Neto A, Demarzo S, Caruso P, Pastore L Jr, et al. Effect of acute kidney injury on weaning from mechanical ventilation in critically ill patients. Crit Care Med. 2007 Jan;35(1):184-91.

23 Epstein SW, Inouye T, Robson M, Oreopoulos DG. Effect of peritoneal dialysis fluid on ventilatory function. Perit Dial Bull. 1982;2: $120-2$.

24 Almeida CP, Ponce D, de Marchi AC, Balbi AL. Effect of peritoneal dialysis on respiratory mechanics in acute kidney injury patients. Perit Dial Int. 2014;34(5):544-9.

25 Almeida CP, Balbi AL, Ponce D. Effect of peritoneal dialysis vs. Haemodialysis on respiratory mechanics in acute kidney injury patients. Clin Exp Nephrol. 2018 Dec;22(6): 1420-6.

26 Honore PM, Hoste E, Molnár Z, Jacobs R, Joannes-Boyau O, Malbrain MLNG, et al. Cytokine removal in human septic shock: where are we and where are we going? Ann Intensive Care. 2019 May 14;9(1):56.

27 Altmann C, Ahuja N, Kiekhaefer CM, Andres Hernando A, Okamura K, Bhargava R, et al. Early peritoneal dialysis reduces lung inflammation in mice with ischemic acute kidney injury. Kidney Int. 2017 Aug;92(2):365-76.

28 Zhao WX, Liu XM, Yu CM, Xu H, Dai JR, Chen HY, et al. Peritoneal dialysis effectively removes toxic substances and improves liver functions of liver failure patients. Eur Rev Med Pharmacol Sci. 2018 Apr;22(8):2432-8.
29 Bokesch PM, Kapural MB, Mossad EB, Cavaglia M, Appachi E, Drummond-Webb JJ, et al. Do peritoneal catheters remove pro-inflammatory cytokines after cardiopulmonary bypass in neonates? Ann Thorac Surg. 2000 Aug;70(2):639-43.

30 Scholten EL, Beitler JR, Prisk GK, Malhotra A. Treatment of ARDS with prone positioning. Chest. 2017 Jan;151(1):215-24.

31 Durand PY, Chanliau J, Gamberoni J, Hestin D, Kessler M. APD: clinical measurement of the maximal acceptable intraperitoneal volume. Adv Perit Dial. 1994;10:63-7.

32 Klisnick A, Souweine B, Filaire M, Wauquier JP, Gazuy N, Deteix P, et al. Peritoneal dialysis in a patient receiving mechanical ventilation in prone position. Perit Dial Int. 1998 SepOct;18(5):536-8.

33 Sourial MY, Sourial MH, Dalsan R, Graham J, Ross M, Chen W, et al. Urgent peritoneal dialysis in patients with COVID-19 and acute kidney injury: a single-center experience in a time of crisis in the United States. Am J Kidney Dis. 2020 Jun 11;

34 Cairns H, Bowes E, Naljayan M. Use of PD for COVID-19-Associated AKI: Clinical Experience and Updated 2020 ISPD Guidelines. 2020. : https://academy.theisn.org/isn/2020/ covid-/2932 75/edwina.brown.brett.cullis. hugh.cairns.elaine.bowes.mihran.naljayan. simon.html? f1/4menu\%3D 13*browseby\% 3D8*s ortby\%3D2*label\%3D19791.

35 Srivatana V, Aggarwal V, Finkelstein FO, Naljayan $\mathrm{M}$, John $\mathrm{H}$, et al. Peritoneal dialysis for acute kidney injury treatment in the United States: brought to you by the COVID-19 pandemic. Kidney360. 2020 May;1(5):410-5.

36 Nagatomo M, Yamada H, Shinozuka K, Shimoto M, Yunoki T, Ohtsuru S. Peritoneal dialysis for COVID-19-associated acute kidney injury. Crit Care. 2020;24(1):309.
37 Abdel-Aal AK, Dybbro P, Hathaway P, Guest S, Neuwirth M, Krishnamurthy V. Best practices consensus protocol for peritoneal dialysis catheter placement by interventional radiologists. Perit Dial Int. 2014;34(5):481-93.

38 Al-Hwiesh AK. Percutaneous peritoneal dialysis catheter insertion by a nephrologist: a new, simple, and safe technique. Perit Dial Int. 2014;34(2):204-11.

39 Li PK, Szeto CC, Piraino B, de Arteaga J, Fan $\mathrm{S}$, Figueiredo AE, et al. ISPD peritonitis recommendations: 2016 update on prevention and treatment. Perit Dial Int. 2016;36(5):481508.

40 International Society for Peritoneal Dialysis. Strategies regarding COVID-19 in PD patients, Adapted from Peking University First Hospital. 2020. : https://ispd.org/strategiescovid19

41 Centers for Disease Control and Prevention. Strategies for optimizing the supply of PPE. CDC. 2020. : https://www.cdc.gov/ coronavirus/2019-ncov/hcp/ppe-strategy/index.html.

42 Cullis B, Abdelraheem M, Abrahams G, Balb A, Cruz DN, Frishberg Y, et al. Peritoneal dialysis for acute kidney injury. Perit Dial Int. 2014 Jul-Aug;34(5):494-517.

43 Ponce D, Balbi AL, Durand JB, Moretta G Divino-Filho JC. Acute peritoneal dialysis in the treatment of COVID-19-related acute kidney injury. Clin Kidney J. 2020;13(3):26973.

44 Durand PY, Chanliau J, Gambéroni J, Hestin D, Kessler M. Measurement of hydrostatic intraperitoneal pressure: a necessary routine test in peritoneal dialysis. Perit Dial Int. 1996; 16(Suppl 1):S84-7. 